PROCEEDINGS OF THE

AMERICAN MATHEMATICAL SOCIETY

Volume 138, Number 2, February 2010, Pages 623-627

S 0002-9939(09)10097-7

Article electronically published on September 16, 2009

\title{
A TETRACHOTOMY FOR CERTAIN ALGEBRAS CONTAINING THE DISC ALGEBRA
}

ALEXANDER J. IZZO

(Communicated by Mario Bonk)

Dedicated to the memory of Juha Heinonen

\begin{abstract}
Let $D$ denote the open unit disc in the complex plane, and let $A(D)$ denote the disc algebra. The algebras $A(D)[f, \bar{f}] \cap C(\bar{D})$, where $f$ is in $H^{\infty}(D)$, are characterized. This answers a question raised by Joseph Cima.
\end{abstract}

\section{INTRODUCTION}

In this paper we answer a question raised by Joseph Cima concerning certain algebras containing the disc algebra. Let $D$ denote the open unit disc in the complex plane, let $C(\bar{D})$ denote the algebra of all complex-valued continuous functions on $\bar{D}$, let $A(D)$ denote the disc algebra (the algebra of holomorphic functions on $D$ that extend continuously to $\bar{D}$ ), and let $H^{\infty}(D)$ denote the algebra of bounded holomorphic functions on $D$. A theorem of E. M. Cirka 2, specialized to the disc, asserts that if $f$ is a function in $C(\bar{D})$ and $f$ is harmonic but not holomorphic on $D$, then the uniformly closed subalgebra $A(D)[f]$ of $C(\bar{D})$ generated by $A(D)$ and $f$ is equal to $C(\bar{D})$. An analogous result for $H^{\infty}(D)$ was proved by Sheldon Axler and Allen Shields [1: If $f$ is a bounded function on $D$ that is harmonic but not holomorphic, then the uniformly closed subalgebra $H^{\infty}(D)[f]$ of $L^{\infty}(D)$ generated by $H^{\infty}(D)$ and $f$ contains $C(\bar{D})$.

Taken together these theorems of Čirka and Axler and Shields lead one to wonder whether the inclusion $A(D)[f] \supset C(\bar{D})$ holds whenever $f$ is a bounded harmonic nonholomorphic function on $D$. In 5 the author showed that this is not the case; in fact it is not even true that $A(D)[f, \bar{f}] \supset C(\bar{D})$ whenever $f \in H^{\infty}(D)$ [5. Theorem 7.6]. However, in [5, Section 7] and 6, the author gave conditions under which the inclusions $A(D)[f] \supset C(\bar{D})$ and $A(D)[f, \bar{f}] \supset C(\bar{D})$ do hold. The author presented these results at the Joint Mathematics Meetings of the American Mathematical Society and the Mathematical Association of America in 2005. At the end of the talk, Joseph Cima raised the question of which continuous functions are in $A(D)[f]$ or $A(D)[f, \bar{f}]$ when the inclusion $A(D)[f] \supset C(\bar{D})$ or $A(D)[f, \bar{f}] \supset C(\bar{D})$ fails. The question was later raised again by Steven Seubert when the author presented the above-mentioned results in a seminar talk at Bowling Green State University.

Received by the editors May 4, 2009.

2000 Mathematics Subject Classification. Primary 46J10, 46J15, 30H05.

(C)2009 American Mathematical Society 
The question of which continuous functions are in $A(D)[f]$ when $f$ is a bounded harmonic function on $D$ appears difficult, and perhaps it is not even possible to give a satisfactory description. However, a crisp answer can be given for $A(D)[f, \bar{f}]$ when $f$ is in $H^{\infty}(D)$, and the present paper is devoted to presenting this answer. The result is as follows.

Theorem 1.1. Let $f \in H^{\infty}(D)$. Then the algebra $A(D)[f, \bar{f}] \cap C(\bar{D})$ is one of the following:

(i) $C(\bar{D})$,

(ii) $\{g \in C(\bar{D}): g|\partial D \in A(D)| \partial D\}$,

(iii) $\{g \in C(\bar{D}): g|(\partial D \cup E) \in A(D)|(\partial D \cup E)\}$, where $\emptyset \subsetneq E \subsetneq D$, or

(iv) $A(D)$.

Note that there are four different possibilities for the algebra $A(D)[f, \bar{f}] \cap C(\bar{D})$, hence the word "tetrachotomy" in the title of the paper. Of course the last three possibilities could be merged into one; taking $E=\emptyset$ in (iii) gives (ii), and taking $E=D$ gives (iv).

Theorem 1.1 will be proven in the next section. The proof we will give yields the following more refined result, which allows one to determine, for a particular function $f \in H^{\infty}(D)$, which of the four possibilities occurs. In the statement of the theorem, $C_{z}$ denotes the cluster set of the function $f$ at the point $z$. (The cluster set of a function $f \in H^{\infty}(D)$ at a point $z \in \partial D$ is defined as follows. Let $\Delta(z, r)$ denote the open disc with center $z$ and radius $r$. Then the cluster set of $f$ at $z$ is $C_{z}=\bigcap_{r>0} \overline{f(D \cap \Delta(z, r))}$.

Theorem 1.2. Let $f \in H^{\infty}(D)$. Let $E=\left\{z \in D: f(z) \in \bigcap_{z \in \partial D} C_{z}\right\}$. If $\bigcap_{z \in \partial D} C_{z}=\emptyset$, then $A(D)[f, \bar{f}] \cap C(\bar{D})=C(\bar{D})$. If $\bigcap_{z \in \partial D} C_{z} \neq \emptyset$, then $A(D)[f, \bar{f}] \cap C(\bar{D})=\{g \in$ $C(\bar{D}): g|(\partial D \cup E) \in A(D)|(\partial D \cup E)\}$.

After the proof of Theorem 1.2, we use the theorem to give a simple proof of an earlier result of the author giving a sufficient condition for the inclusion $A(D)[f, \bar{f}] \supset C(\bar{D})$, and we show that the sufficient condition is not necessary. We also give examples to show that each of the four cases in Theorem 1.1 really does occur.

This paper was completed while the author was a visitor at the Institute for Mathematics and its Applications at the University of Minnesota. He would like to thank the institute for its hospitality.

\section{PRoOF AND EXAMPLES}

Before beginning the proof of Theorem 1.2 (from which Theorem 1.1 follows immediately), we make explicit some conventions already used in the introduction. The algebras we are dealing with are really algebras of bounded continuous functions on the open unit disc. Thus we tacitly identify $C(\bar{D})$ with the algebra of uniformly continuous functions on $D$ by identifying each member of $C(\bar{D})$ with its restriction to $D$. Given bounded continuous complex-valued functions $f_{1}, \ldots, f_{n}$ on $D$, we denote by $A(D)\left[f_{1}, \ldots, f_{n}\right]$ the uniformly closed algebra on $D$ generated by $A(D)$ and the functions $f_{1}, \ldots, f_{n}$. Given a collection $A$ of functions on a space $X$ and a subset $S$ of $X$, we denote by $A \mid S$ the collection of functions obtained by restricting the functions in $A$ to $S$. 
Proof of Theorem 1.2. Let $X=\{(z, f(z), \bar{f}(z)): z \in D\} \cup\{(z, w, \bar{w}): z \in \partial D, w \in$ $\left.C_{z}\right\}$. Note that $X$ is the closure of the set $\{(z, f(z), \bar{f}(z)): z \in D\}$ in $\mathbb{C}^{3}$ and that $X$ is compact. Also note that if we identify $D$ with $\{(z, f(z), \bar{f}(z)): z \in D\}$, then every function in $A(D)[f, \bar{f}]$ extends uniquely to a continuous function on $X$. Thus we can regard $A(D)[f, \bar{f}]$ as a uniform algebra on $X$, and it will be helpful to do so. The functions in $C(\bar{D})$ also extend uniquely to $X$, so we can regard $C(\bar{D})$ as an algebra on $X$ as well. Note that then $C(\bar{D})$ consists of the continuous functions on $X$ that are constant on the fibers of the map $\pi: X \rightarrow \bar{D}$ given by $\pi\left(z_{1}, z_{2}, z_{3}\right)=z_{1}$.

We will prove the theorem by considering the maximal sets of antisymmetry for $A(D)[f, \bar{f}]$ (regarded as a uniform algebra on $X$ ) and applying the Bishop antisymmetric decomposition [3, Theorem II.13.1]. Since both $f$ and $\bar{f}$ are in the algebra, the real and imaginary parts of $f$ are in the algebra. Thus $f$ (regarded as a function on $X$ ) must be constant on each set of antisymmetry. Hence each set of antisymmetry lies in a level set of $f$. Consequently, each set of antisymmetry is the union of a (possibly empty) discrete subset of $D$ with a (possibly empty) set lying over $\partial D$.

Consider the case when the set $\bigcap_{z \in \partial D} C_{z}$ is empty. Let $g \in C(\bar{D})$ be arbitrary, and let $K$ be a maximal set of antisymmetry for $A(D)[f, \bar{f}]$. The assumption that $\bigcap C_{z}=\emptyset$ implies that $\pi(K)$ does not contain the full circle $\partial D$. Consequently, $A(D) \mid \pi(K)$ is dense in $C(\pi(K))$ (by Laurentiev's theorem [3, Theorem II.8.7] for instance). Hence viewing the functions as defined on $X$, we have that $g \mid K$ is a uniform limit of functions in $A(D)$. Since the restriction of a uniform algebra to a maximal set of antisymmetry is always closed, we get that $g \mid K$ is in $A(D)[f, \bar{f}] \mid K$. Now the Bishop antisymmetric decomposition gives that $g \in A(D)[f, \bar{f}]$. We conclude that $A(D)[f, \bar{f}] \supset C(\bar{D})$, as desired.

Now consider the case when the set $\bigcap_{z \in \partial D} C_{z}$ is nonempty. Let $E=\{z \in D$ : $\left.f(z) \in \bigcap_{z \in \partial D} C_{z}\right\}$. We will show that $A(D)[f, \bar{f}] \cap C(\bar{D})=\{g \in C(\bar{D}): g \mid(\partial D \cup E) \in$ $A(D) \mid(\partial D \cup E)\}$ as asserted in the theorem.

Let $g \in C(\bar{D})$ be such that $g|(\partial D \cup E) \in A(D)|(\partial D \cup E)$, and let $K$ be a maximal set of antisymmetry for $A(D)[f, \bar{f}]$. Let $\lambda$ be the value of $f$ on $K$. If $\lambda \notin \bigcap_{z \in \partial D} C_{z}$, then $\pi(K)$ does not contain the full circle $\partial D$. Consequently, $g|K \in A(D)[f, \bar{f}]| K$ by the same reasoning as that used above in the case when $\bigcap_{z \in \partial D} C_{z}=\emptyset$. If $\lambda \in$ $\bigcap C_{z}$, then $K$ is contained in $\pi^{-1}(\partial D) \cup E$. By assumption there is a function in $z \in \partial D$

$A(D)$ that agrees with $g$ on $\partial D \cup E$. Consequently, when we regard the functions as defined on $X$, there is a function in $A(D)$ that agrees with $g$ on $\pi^{-1}(\partial D) \cup E$ and hence in particular on $K$. Applying the Bishop antisymmetric decomposition, we conclude that $g$ is in $A(D)[f, \bar{f}]$. Thus $A(D)[f, \bar{f}] \cap C(\bar{D}) \supset\{g \in C(\bar{D})$ : $g|(\partial D \cup E) \in A(D)|(\partial D \cup E)\}$.

For the reverse inclusion suppose now that $h \in A(D)[f, \bar{f}] \cap C(\bar{D})$. Let $\lambda$ be a point in $\bigcap_{z \in \partial D} C_{z}$ and let $K_{\lambda}=\{x \in X: f(x)=\lambda\}$. Then $\pi\left(K_{\lambda}\right)$ contains $\partial D$. Since $f$ is constant on $K_{\lambda}$, we have that $A(D) \mid K_{\lambda}$ is dense in $A(D)[f, \bar{f}] \mid K_{\lambda}$. But since $\pi\left(K_{\lambda}\right)$ contains $\partial D$, every function in $A(D)$ attains its maximum modulus on $K_{\lambda}$. Thus $A(D) \mid K_{\lambda}$ is closed, so $A(D)\left|K_{\lambda}=A(D)[f, \bar{f}]\right| K_{\lambda}$. Thus $h$ agrees with an 
element of $A(D)$ on $K_{\lambda}$. In particular, $h$ agrees with an element $p$ of $A(D)$ on $\partial D$. Now let $z \in E$ be arbitrary and let $\zeta=f(z)$. By the argument just given, $h \mid K_{\zeta}$ must agree with an element of $A(D)$ on $K_{\zeta}$. But since $K_{\zeta}$ contains a point in each fiber over $\partial D$, that element of $A(D)$ must be $p$. Thus $h$ agrees with $p$ on $\partial D \cup E$. We conclude that $A(D)[f, \bar{f}] \cap C(\bar{D}) \subset\{g \in C(\bar{D}): g|(\partial D \cup E) \in A(D)|(\partial D \cup E)\}$.

As a corollary of Theorem 1.2 we can re-prove the following earlier result of the author [5, Theorem 7.7], giving a sufficient condition for the inclusion $A(D)[f, \bar{f}] \supset$ $C(\bar{D})$. Note that here we obtain the result by a route that is incomparably simpler than the one originally used in 5 .

Corollory 2.1 ([5). If $f \in H^{\infty}(D)$ is nonconstant and is continuous on a subset of $\partial D$ of positive (one-dimensional Lebesgue) measure, then $A(D)[f, \bar{f}] \supset C(\bar{D})$.

Proof. At each point $z \in \partial D$ where $f$ is continuous, the cluster set $C_{z}$ consists of the single point $f(z)$. Let $S$ be the subset of $\partial D$ on which $f$ is continuous. Since $S$ has positive measure, $f$ cannot be constant on $S$, for otherwise $f$ would be constant on $D$ by a well-known theorem [7, Theorem 17.18]. Consequently, $\bigcap_{z \in \partial D} C_{z}=\emptyset$, so $A(D)[f, \bar{f}] \supset C(\bar{D})$ by Theorem 1.2 .

In 6] the author gave a partial converse to the above corollary by showing that for every subset $S$ of $\partial D$ of measure zero, there is a function $f \in H^{\infty}(D)$ that is continuous on $S$ and such that the inclusion $A(D)[f, \bar{f}] \supset C(\bar{D})$ fails. We now give an example to show that the full converse to the above corollary is false; that is, we show that there is a function $f \in H^{\infty}(D)$ that fails to be continuous on a set of positive measure but for which the inclusion $A(D)[f, \bar{f}] \supset C(\bar{D})$ holds. In fact our example shows that $f$ can even be discontinuous everywhere on $\partial D$.

Example 1. Let $h$ be an inner function on $D$ that is discontinuous everywhere on $\partial D$. Let $f=h+2 z$. Then $f \in H^{\infty}(D)$ and is discontinuous everywhere on $\partial D$, but $\bigcap_{z \in \partial D} C_{z}=\emptyset$ as every point in the cluster set $C_{1}$ has real part $\geq 1$, while every point in $C_{-1}$ has real part $\leq-1$. Hence $A(D)[f, \bar{f}] \supset C(\bar{D})$, by Theorem 1.2.

We conclude the paper by presenting examples showing that each of the four cases in Theorem 1.1 really does occur. We have just seen that case (i) occurs. We consider the remaining cases in the order (iv), (ii), (iii).

Example 2. Let $f$ be an inner function on $D$ that is discontinuous everywhere on $\partial D$. Then by [4, Theorem II.6.6] the cluster set of $f$ at each point of $\partial D$ is $\bar{D}$, so $\bigcap C_{z}=\bar{D}$. Consequently, Theorem 1.2 shows that $A(D)[f, \bar{f}] \cap C(\bar{D})=C(\bar{D})$; $z \in \partial D$

i.e., case (iv) of Theorem 1.1 holds.

Example 3. Let $s$ be a singular inner function that is discontinuous everywhere on $\partial D$. Let $f=\left(\frac{1+z}{2}\right) s$. Then $f$ is continuous at -1 and takes the value 0 there, so $\bigcap C_{z}=\{0\}$. Since $f$ has no zeros on $D$, Theorem 1.2 gives $A(D)[f, \bar{f}] \cap C(\bar{D})=$ $z \in \partial D$

$\{g \in C(\bar{D}): g|\partial D \in A(D)| \partial D\}$; i.e., case (ii) of Theorem 1.1 holds.

Example 4. The preceding example can be generalized to show that for each subset $S$ of $\partial D$ of measure zero there is an $H^{\infty}$ function that is continuous on $S$ such that case (ii) of Theorem 1.1 holds. As before, let $s$ be a singular inner 
function that is discontinuous everywhere on $\partial D$. By [6, Theorem 1.7] there is a nonzero function $h$ in $H^{\infty}(D)$ that is continuous on $S$ and is zero at every point of $S$. Furthermore, the proof in [6] shows that $h$ can be taken to have no zeros in $D$. Let $f=h \cdot s$. Then $f$ is continuous on $S$ and is zero at every point of $S$, and $f$ has no zeros in $D$. Applying Theorem 1.2 gives that case (ii) of Theorem 1.1 holds for $f$.

Example 5. By modifying the preceding example, we show that for each subset $S$ of $\partial D$ of measure zero there is an $H^{\infty}$ function that is continuous on $S$ such that case (iii) of Theorem 1.1 holds. Let $f$ be as in the preceding example and let $w \in A(D)$ be a nonconstant function with some zeros on $D$. Let $E=\{z \in D: w(z)=0\}$. Set $k=f \cdot w$. Then $\bigcap_{z \in \partial D} C_{z}(k)=\{0\}$, where $C_{z}(k)$ is the cluster set for $k$ at $z$. So Theorem 1.2 gives $A(D)[k, \bar{k}] \cap C(\bar{D})=\{g \in C(\bar{D}): g|(\partial D \cup E) \in A(D)|(\partial D \cup E)\}$, where $\phi \subsetneq E \subsetneq D$; i.e., case (iii) of Theorem 1.1 holds.

Example 6. In the preceding example, the set $E$ is discrete. We now give an example where the set $E$ has interior. Let $h$ be an inner function that is discontinuous everywhere on $\partial D$ and let $f=(1+2 z) h$. Then $\bigcap_{z \in \partial D} C_{z}=\bar{D}$. Let $E=\{z \in D$ :

$f(z) \in \bar{D}\}$. Then $E$ is a proper subset of $D$ having (nonempty) interior and by Theorem 1.2, $A(D)[f, \bar{f}] \cap C(\bar{D})=\{g \in C(\bar{D}): g|(\partial D \cup E) \in A(D)|(\partial D \cup E)\}$; i.e., case (iii) of Theorem 1.1 holds.

As a final observation, note that while cases (ii) and (iii) can occur for functions that are continuous on a subset of $\partial D$ of measure zero, case (iv) occurs only for functions that are discontinuous everywhere.

\section{REFERENCES}

[1] S. Axler and A. Shields, Algebras generated by analytic and harmonic functions, Indiana Univ. Math. J. 36 (1987), 631-638. MR905614 (88h:46102)

[2] E. M. Čirka, Approximation by holomorphic functions on smooth manifolds in $\mathbb{C}^{n}$, Mat. Sb. (N.S.) 78 (1969), 101-123; English transl., Math. USSR Sb. 7 (1969), 95-114. MR0239121 $(39: 480)$

[3] T. W. Gamelin, Uniform Algebras, 2nd ed., Chelsea, New York, 1984.

[4] J. B. Garnett, Bounded Analytic Functions, Academic Press, New York, 1981. MR628971 (83g:30037)

[5] A. J. Izzo, Algebras containing bounded holomorphic functions, Indiana Univ. Math. J. 52 (2003), 1305-1342. MR2010729 (2004h:46055)

[6] A. J. Izzo, Algebras generated by the disc algebra and bounded harmonic functions, Proc. Amer. Math. Soc. 135 (2007), 1065-1071. MR2262907(2007k:46087)

[7] W. Rudin, Real and Complex Analysis, 3rd ed., McGraw-Hill, New York, 1987. MR.924157 (88k:00002)

Department of Mathematics and Statistics, Bowling Green State University, Bowling Green, Ohio 43403

E-mail address: aizzo@math.bgsu.edu 